\title{
The Laplacian Spectral Radius of a Class of Unicyclic Graphs
}

\author{
Haixia Zhang \\ Department of Mathematics, Taiyuan University of Science and Technology, Taiyuan 030024, China \\ Correspondence should be addressed to Haixia Zhang; zhanghaixiass@hotmail.com
}

Received 30 June 2013; Accepted 25 November 2013

Academic Editor: Ram N. Mohapatra

Copyright (C) 2013 Haixia Zhang. This is an open access article distributed under the Creative Commons Attribution License, which permits unrestricted use, distribution, and reproduction in any medium, provided the original work is properly cited.

Let $C(n, k)$ be the set of all unicyclic graphs with $n$ vertices and cycle length $k$. For any $U \in C(n, k), U$ consists of the (unique) cycle (say $C_{k}$ ) of length $k$ and a certain number of trees attached to the vertices of $C_{k}$ having (in total) $n-k$ edges. If there are at most two trees attached to the vertices of $C_{k}$, where $k$ is even, we identify in the class of unicyclic graphs those graphs whose Laplacian spectral radii are minimal.

\section{Introduction}

Following [1], let $G=(V, E)$ be a simple connected graph on $n$ vertices and $m$ edges (so $n=|V(G)|$ is its order and $m=$ $|E(G)|$ is its size). For $v_{i} \in V$, the degree of $v_{i}$ is denoted by $d_{v_{i}}$. Let $\Delta(G)$ be the maximum degree of $G$. For two vertices $v_{i}$ and $v_{j}(i \neq j)$ in $G$, the distance between $v_{i}$ and $v_{j}$, denoted by $d\left(v_{i}, v_{j}\right)$, is the number of edges in a shortest path joining $v_{i}$ and $v_{j}$.

Let $C(n, k)$ be the set of all unicyclic graphs on $n$ vertices and the cycle length $k$. So, if $U \in C(n, k)$, then $U$ consists of the (unique) cycle (say $C_{k}$ ) of length $k$ and a certain number of trees attached to vertices of $C_{k}$ having (in total) $n-k$ edges. If the cycle length of a unicyclic graph is even (odd), we call it an even (odd) unicyclic graph. We may assume that vertices of $C_{k}$ are $c_{1}, c_{2}, \ldots, c_{k}$ or for short only $1,2, \ldots, k$ (ordered in a natural way around $C_{k}$, say, in the clockwise direction). For each $i$, let $T_{i}$ be a rooted tree (with $r_{i}$ as its root) attached to $c_{i}$. Then, for each $U \in C(n, k)$, we can write $U=C\left(T_{1}, T_{2}, \ldots, T_{k}\right)$. If $T_{i}$, for each $i$, is a path $P_{m_{i}+1}$, whose root is a vertex of minimum degree, then we write $U=P\left(m_{1}, m_{2}, \ldots, m_{k}\right)$.

Let $A(G)$ be the adjacency matrix of $G$ and $D(G)=$ $\operatorname{diag}\left(d_{v_{1}}, d_{v_{2}}, \ldots, d_{v_{n}}\right)$ the diagonal matrix. Then the Laplacian matrix of $G$ is $L(G)=D(G)-A(G)$. Since $L(G)$ is real symmetric and positive semidefinite, its eigenvalues are nonnegative real numbers. For a graph $G$, we denote by $\mu_{1}(G)$ the largest eigenvalue of $L(G)$ and call it the Laplacian spectral radius.
The investigation on the Laplacian spectral radius of graphs is an important topic in the theory of graph spectra. Since 1980s, there are several monographs and a lot of research papers published continually (see [2-7]). Recently, the problem concerning graphs with maximal or minimal Laplacian spectral radius of a given class of graphs has been studied by many authors. Guo [8] determined the first four graphs with the largest Laplacian spectral radius among all unicyclic graphs on $n$ vertices.

In this paper, for any $U \in C(n, k)$, if there are at most two trees attached to vertices of $C_{k}$, where $k$ is even, we characterize in the class of even unicyclic graphs those graphs whose Laplacian spectral radii are minimal.

\section{Main Results and Proofs}

Let $L_{v_{i}}(G)$ be the principal submatrix obtained from $L(G)$ by deleting the corresponding row and column of $v_{i}$. Generally, let $L_{S}(G)$ be the principal submatrix obtained from $L(G)$ by deleting the corresponding rows and columns of all vertices of $S$. For any square matrix $B$, denote by $\Phi(B)=\Phi(B, x)=$ $\operatorname{det}(x I-B)$ the characteristic polynomial of $B$. In particular, if $B=L(G)$, we write $\Phi(L(G))$ by $\Phi(G)$ for convenience. If $G=u$, then suppose that $\Phi\left(L_{u}(G)\right)=1$.

Lemma 1 (see [9]). Let $G_{1}$ and $G_{2}$ be two graphs. If $\Phi\left(G_{1}\right)>$ $\Phi\left(G_{2}\right)$ for $x \geq \mu_{1}\left(G_{2}\right)$, then $\mu_{1}\left(G_{1}\right)<\mu_{1}\left(G_{2}\right)$.

Lemma 2 (see [4]). Let $G$ be a graph containing at least one edge. Then $\mu_{1}(G) \geq \Delta(G)+1$. Moreover, if $G$ is connected on $n>1$ vertices, the equality holds if and only if $\Delta(G)=n-1$. 
An internal path of a graph $G$ is a sequence of vertices $v_{1}, v_{2}, \ldots, v_{k}$ with $k \geq 2$ such that

(1) the vertices in the sequence are distinct (except possibly $v_{1}=v_{k}$ );

(2) $v_{i}$ is adjacent to $v_{i+1},(i=1,2, \ldots, k-1)$;

(3) the vertex degrees $d_{v_{i}}$ satisfy $d_{v_{1}} \geq 3, d_{v_{2}}=\cdots=$ $d_{v_{k-1}}=2$ (unless $k=2$ ), and $d_{v_{k}} \geq 3$.

Lemma 3 (see [10]). Let $G$ be a connected bipartite graph with vertex set $V(G)=\left\{v_{1}, v_{2}, \ldots, v_{n}\right\}$ and $u v$ an edge on an internal path $P: v_{1} v_{2} \cdots v_{k}(n>k \geq 2)$ of $G$. Let $G_{2 l+1}(l \geq 1)$ be the graph obtained from $G$ by subdividing the edge uv into $2 l+1$ new edges. Then one has $\mu_{1}(G)>\mu_{1}\left(G_{2 l+1}\right)>\mu_{1}\left(G_{2 l+3}\right)$.

Let $v$ be a vertex of a connected graph $G$ with at least two vertices. Let $G_{k, l}(l \geq k \geq 1)$ be the graph obtained from $G$ by attaching two new paths $P: v\left(=v_{0}\right) v_{1} v_{2} \cdots v_{k}$ and $Q$ : $v\left(=v_{0}\right) u_{1} u_{2} \cdots u_{l}$ of length $k$ and $l$, respectively, at $v$, where $u_{1}, u_{2}, \ldots, u_{l}$ and $v_{1}, v_{2}, \ldots, v_{k}$ are distinct new vertices. Let $G_{k-1, l+1}=G_{k, l}-v_{k-1} v_{k}+u_{l} v_{k}$.

Lemma 4 (see [11]). If $G$ is a connected bipartite graph on $n \geq$ 2 vertices and $v$ is a vertex of $G$, let $G_{k, l}$ be the graph defined as above. If $l \geq k \geq 1$, then $\mu_{1}\left(G_{k-1, l+1}\right)<\mu_{1}\left(G_{k, l}\right)$.

Lemma 5 (see [12]). Let $D_{n}(n \geq 1)$ be the matrix obtained from $L\left(P_{n+2}\right)$ by deleting the rows and columns corresponding to two end vertices of $P_{n+2}$, and suppose that $\Phi\left(D_{0}\right)=1$, $\Phi\left(D_{-n}\right)=0$; then

(i) $x \Phi\left(D_{n-1}\right)=\Phi\left(P_{n}\right)$;

(ii) $\Phi\left(D_{n+1}\right)=(x-2) \Phi\left(D_{n}\right)-\Phi\left(D_{n-1}\right)$;

(iii) $\Phi\left(D_{m+1}\right) \Phi\left(D_{n}\right)-\Phi\left(D_{m}\right) \Phi\left(D_{n+1}\right)=\Phi\left(D_{m}\right) \Phi\left(D_{n-1}\right)-$ $\Phi\left(D_{m-1}\right) \Phi\left(D_{n}\right),(n, m \geq 1)$;

(iv) $\Phi\left(C_{n}\right)=\Phi\left(D_{n}\right)-\Phi\left(D_{n-2}\right)+2(-1)^{n+1}$.

From Lemma 5(i), all eigenvalues of $D_{n}$ are $2+$ $2 \cos (i \pi /(n+1))$, where $1 \leq i \leq n$. Other characterizations of $\Phi\left(D_{n}\right)$ can be shown as follows.

Lemma 6 (see [9]). Let $\Phi\left(D_{m}\right) \Phi\left(D_{n}\right)-\Phi\left(D_{m-1}\right) \Phi\left(D_{n+1}\right)=$ $\Phi\left(D_{n-m}\right)$, where $0 \leq m \leq n$.

Lemma 7. For $n, m \geq 1, \Phi\left(D_{n+m}\right)=\Phi\left(D_{n}\right) \Phi\left(D_{m}\right)-$ $\Phi\left(D_{n-1}\right) \Phi\left(D_{m-1}\right)$.

Proof. It is easily obtained from some properties of the determinant $\Phi\left(D_{n}\right)$.

In the following, we give a transformation property of unicyclic graphs.

Lemma 8. Let $u(=i)$ and $v(=j)$ be two nonadjacent vertices of the cycle $C_{k}$, and suppose that $d(u, v)=a+1$; that is, $a$ is the number of vertices in the shortest path $P_{a+2}$, not including $u$ and $v$, where $1 \leq a \leq\lfloor(k-2) / 2\rfloor$. Let $G_{a}(m, n)=$ $P\left(m_{1}, m_{2}, \ldots, m_{k}\right)$, where only $m_{i}=m$ and $m_{j}=n(1 \leq$ $i, j \leq k)$ are nonzero. If $m, n \geq 1$, then $\mu_{1}\left(G_{a}(m, n)\right)<$ $\mu_{1}\left(G_{a-1}(m, n)\right)$.
Proof

Case $1(n>2)$. Label vertices of $G_{a}(m, n)$ properly; we have

$$
\begin{aligned}
& \Phi\left(G_{a}(m, n)\right) \\
& \quad=\left|\begin{array}{cccc}
x I-L_{v_{1}}\left(G_{a}(m, 1)\right) & e_{1} & O & O \\
e_{1}^{T} & x-2 & 1 & O \\
O & 1 & x-2 & f_{1}^{T} \\
O & O & f_{1} & x I-L_{v_{2}}\left(P_{n-1}\right)
\end{array}\right|,
\end{aligned}
$$

where $v_{1}, v_{2} \in V\left(P_{n+1}\right)$ and $v_{1}$ and $v_{2}$ are adjacent to $v$ and $v_{1}$, respectively. $e_{1}$ is the $(k+m) \times 1$ column vector whose only nonzero entry is 1 in the $(m+a+2)$ th position, and $f_{1}$ is the $(n-2) \times 1$ column vector whose only nonzero entry is 1 in the first position.

By a direct computation, we can obtain

$$
\Phi\left(G_{a}(m, n)\right)=\operatorname{det}(A) \operatorname{det}(B)-\operatorname{det}\left(A^{\prime}\right) \operatorname{det}\left(B^{\prime}\right),
$$

where

$$
\begin{gathered}
\operatorname{det}(A)=\left|\begin{array}{rr}
x I-L_{v_{1}}\left(G_{a}(m, 1)\right) & e_{1} \\
e_{1}^{T} & x-2
\end{array}\right|, \\
\operatorname{det}\left(A^{\prime}\right)=\operatorname{det}\left(x I-L_{v_{1}}\left(G_{a}(m, 1)\right)\right), \\
\operatorname{det}(B)=\left|\begin{array}{cc}
x-2 & f_{1}^{T} \\
f_{1} & x I-L_{v_{2}}\left(P_{n-1}\right)
\end{array}\right|=\Phi\left(D_{n-1}\right)+\Phi\left(D_{n-2}\right), \\
\operatorname{det}\left(B^{\prime}\right)=\operatorname{det}\left(x I-L_{v_{2}}\left(P_{n-1}\right)\right)=\Phi\left(D_{n-2}\right)+\Phi\left(D_{n-3}\right) .
\end{gathered}
$$

Furthermore,

$$
\begin{aligned}
\operatorname{det}(A) & =\left|\begin{array}{ccc}
x I-L_{u_{1}}\left(P_{m}\right) & g_{1} & 0 \\
g_{1}^{T} & x-2 & h_{1}^{T} \\
0 & h_{1} & x I-L_{u_{1}, v_{2}}\left(G_{a}(1,2)\right)
\end{array}\right| \\
& =\operatorname{det}(D) \operatorname{det}(C)-\operatorname{det}\left(D^{\prime}\right) \operatorname{det}\left(C^{\prime}\right),
\end{aligned}
$$

where $u_{1} \in V\left(P_{m+1}\right)$ is adjacent to $u . g_{1}$ is the $(m-1) \times 1$ column vector, whose only nonzero entry is 1 in the last position. $h_{1}$ is the $(k+1) \times 1$ column vector, whose only nonzero entry is 1 in the first position.

Note that

$$
\begin{gathered}
\operatorname{det}(D)=\left|\begin{array}{cc}
x I-L_{u_{1}}\left(P_{m}\right) & g_{1} \\
g_{1}^{T} & x-2
\end{array}\right|=\Phi\left(D_{m}\right)+\Phi\left(D_{m-1}\right), \\
\operatorname{det}\left(D^{\prime}\right)=\operatorname{det}\left(x I-L_{u_{1}}\left(P_{m}\right)\right)=\Phi\left(D_{m-1}\right)+\Phi\left(D_{m-2}\right), \\
\operatorname{det}(C)=\operatorname{det}\left(x I-L_{u_{1}, v_{2}}\left(G_{a}(1,2)\right)\right) \\
=(x-2) \Phi\left(C_{k}\right)-(2 x-3) \Phi\left(D_{k-1}\right) \\
\quad+(x-1) \Phi\left(D_{a}\right) \Phi\left(D_{k-2-a}\right), \\
\operatorname{det}\left(C^{\prime}\right)=\operatorname{det}\left(x I-L_{u, u_{1}, v_{2}}\left(G_{a}(1,2)\right)\right) \\
=(x-2) \Phi\left(D_{k-1}\right)-(x-1) \Phi\left(D_{a}\right) \Phi\left(D_{k-2-a}\right) .
\end{gathered}
$$


Similarly,

$$
\begin{aligned}
& \operatorname{det}\left(A^{\prime}\right) \\
& =\operatorname{det}(D)\left[\Phi\left(C_{k}\right)-2 \Phi\left(D_{k-1}\right)+\Phi\left(D_{a}\right) \Phi\left(D_{k-2-a}\right)\right] \\
& \quad-\operatorname{det}\left(D^{\prime}\right)\left[\Phi\left(D_{k-1}\right)-\Phi\left(D_{a}\right) \Phi\left(D_{k-2-a}\right)\right] .
\end{aligned}
$$

Combing the equations previous, we get

$$
\begin{aligned}
\Phi\left(G_{a}(m, n)\right)= & h(x) \operatorname{det}(D) \operatorname{det}(B)-i(x) \operatorname{det}\left(D^{\prime}\right) \operatorname{det}(B) \\
& -g(x) \operatorname{det}(D) \operatorname{det}\left(B^{\prime}\right) \\
& +k(x) \operatorname{det}\left(D^{\prime}\right) \operatorname{det}\left(B^{\prime}\right),
\end{aligned}
$$

where

$$
\begin{gathered}
h(x)=\operatorname{det}(C), \quad i(x)=\operatorname{det}\left(C^{\prime}\right), \\
k(x)=\Phi\left(D_{k-1}\right)-\Phi\left(D_{a}\right) \Phi\left(D_{k-2-a}\right), \\
g(x)=\Phi\left(C_{k}\right)-2 \Phi\left(D_{k-1}\right)+\Phi\left(D_{a}\right) \Phi\left(D_{k-2-a}\right) .
\end{gathered}
$$

From Lemma 2, $\mu_{1}\left(G_{a-1}(m, n)\right)>4$. By Lemmas 5(ii) and 6 and (7),

$$
\begin{aligned}
\Phi\left(G_{a}(m, n)\right)-\Phi\left(G_{a-1}(m, n)\right) \\
=\Phi\left(D_{k-2-2 a}\right)\left[(x-1) \operatorname{det}(B)-\operatorname{det}\left(B^{\prime}\right)\right] \\
\quad \times\left(\operatorname{det}(D)+\operatorname{det}\left(D^{\prime}\right)\right) \\
=x^{2} \Phi\left(D_{k-2-2 a}\right) \Phi\left(D_{n-1}\right) \Phi\left(D_{m-1}\right)>0,
\end{aligned}
$$

for $x \geq \mu_{1}\left(G_{a-1}(m, n)\right)$.

So $\mu_{1}\left(G_{a}(m, n)\right)<\mu_{1}\left(G_{a-1}(m, n)\right)$ holds from Lemma 1 .

When $n=1,2$, we appropriately modify $\Phi\left(G_{a}(m, n)\right)$ and get the same result.

Next we consider some special even unicyclic graphs $G_{a}(m, n)$.

By Lemmas 6 and 7, we have

(i) $\Phi\left(D_{k-1}\right)=\Phi\left(D_{k / 2}\right) \Phi\left(D_{(k-2) / 2}\right)-\Phi\left(D_{(k-2) / 2}\right)$

$$
\times \Phi\left(D_{(k-4) / 2}\right)
$$

(ii) $\Phi\left(D_{k-2}\right)=\Phi^{2}\left(D_{(k-2) / 2}\right)-\Phi^{2}\left(D_{(k-4) / 2}\right)$,

(iii) $\Phi^{2}\left(D_{(k-2) / 2}\right)-\Phi\left(D_{k / 2}\right) \Phi\left(D_{(k-4) / 2}\right)=1$.

From Lemmas 5(ii) and 5(iv) and (10), we get

$$
\begin{aligned}
\Phi\left(C_{k}\right) & =\Phi\left(D_{k}\right)-\Phi\left(D_{k-2}\right)-2 \\
& =(x-2) \Phi\left(D_{k-1}\right)-2 \Phi\left(D_{k-2}\right)-2 \\
& =x(x-4) \Phi^{2}\left(D_{(k-2) / 2}\right) .
\end{aligned}
$$

By (11) and (i) of (10),

$$
\begin{aligned}
\operatorname{det}(A)=\Phi\left(D_{(k-2) / 2}\right) & \\
\times & \operatorname{det}(D)\left[\left(x^{3}-8 x^{2}+16 x-7\right) \Phi\left(D_{(k-2) / 2}\right)\right. \\
& \left.+2(2 x-3) \Phi\left(D_{(k-4) / 2}\right)\right]-\operatorname{det}\left(D^{\prime}\right) \\
& \times\left[\left(x^{5}-5 x+5\right) \Phi\left(D_{(k-2) / 2}\right)\right. \\
\operatorname{det}\left(A^{\prime}\right)=\Phi( & \left.\left.-2(x-2) \Phi\left(D_{(k-4) / 2}\right)\right]\right\} \\
\times & \left\{\operatorname { d e t } ( D ) \left[\left(x^{2}-6 x+5\right) \Phi\left(D_{(k-2) / 2}\right)\right.\right. \\
& \left.+4 \Phi\left(D_{(k-4) / 2}\right)\right]-\operatorname{det}\left(D^{\prime}\right) \\
& \left.\times\left[(x-3) \Phi\left(D_{(k-2) / 2}\right)-2 \Phi\left(D_{(k-4) / 2}\right)\right]\right\} .
\end{aligned}
$$

Case 1. The unicyclic graph $G_{2 n}(n-1, n)$, where $k=4 n+2$ $(n \geq 2)$, is as follows.

Note that

$$
\begin{aligned}
& \operatorname{det}(D) \operatorname{det}(B)=(x-1)^{2} \Phi^{2}\left(D_{n-2}\right)-2(x-1) \\
& \times \Phi\left(D_{n-2}\right) \Phi\left(D_{n-3}\right)+\Phi^{2}\left(D_{n-3}\right) \\
& \operatorname{det}\left(D^{\prime}\right) \operatorname{det}(B)=(x-1) \Phi^{2}\left(D_{n-2}\right)+(x-2) \Phi\left(D_{n-2}\right) \\
& \times \Phi\left(D_{n-3}\right)-\Phi^{2}\left(D_{n-3}\right) \\
& \operatorname{det}(D) \operatorname{det}\left(B^{\prime}\right)=\operatorname{det}\left(D^{\prime}\right) \operatorname{det}(B) \\
& \operatorname{det}\left(D^{\prime}\right) \operatorname{det}\left(B^{\prime}\right)= \Phi^{2}\left(D_{n-2}\right)+2 \Phi\left(D_{n-2}\right) \Phi\left(D_{n-3}\right) \\
&+\Phi^{2}\left(D_{n-3}\right)
\end{aligned}
$$

In view of (2) and (12)-(13), we have

$$
\begin{gathered}
\Phi\left(G_{2 n}(n-1, n)\right) \\
=x \Phi\left(D_{2 n}\right)\left\{\left(x^{4}-10 x^{3}+31 x^{2}-34 x+10\right) \Phi^{2}\left(D_{n-2}\right)\right. \\
-\left(2 x^{3}-16 x^{2}+33 x-16\right) \Phi\left(D_{n-2}\right) \\
\left.\times \Phi\left(D_{n-3}\right)+\left(x^{2}-6 x+6\right) \Phi^{2}\left(D_{n-3}\right)\right\} \\
+2 x \Phi\left(D_{2 n-1}\right)\left\{\left(2 x^{2}-6 x+3\right) \Phi^{2}\left(D_{n-2}\right)-(3 x-4)\right. \\
\left.\times \Phi\left(D_{n-2}\right) \Phi\left(D_{n-3}\right)+\Phi^{2}\left(D_{n-3}\right)\right\} \\
=x\left(S_{1} \Phi\left(D_{2 n}\right)+2 S_{2} \Phi\left(D_{2 n-1}\right)\right) .
\end{gathered}
$$


By Lemmas 5(ii) and 7,

$$
\begin{aligned}
\Phi\left(D_{2 n}\right) & -2 \Phi\left(D_{2 n-1}\right) \\
= & \Phi^{2}\left(D_{n}\right)-\Phi^{2}\left(D_{n-1}\right) \\
& -2\left(\Phi\left(D_{n}\right) \Phi\left(D_{n-1}\right)-\Phi\left(D_{n-1}\right) \Phi\left(D_{n-2}\right)\right) \\
= & \left(x^{4}-10 x^{3}+33 x^{2}-40 x+13\right) \Phi^{2}\left(D_{n-2}\right) \\
& -\left(2 x^{3}-16 x^{2}+36 x-20\right) \Phi\left(D_{n-2}\right) \Phi\left(D_{n-3}\right) \\
& +\left(x^{2}-6 x+7\right) \Phi^{2}\left(D_{n-3}\right) \\
= & S_{1}+S_{2} .
\end{aligned}
$$

So,

$$
\begin{aligned}
\Phi\left(G_{2 n}(n-1, n)\right) \\
=x\left[\left(\Phi\left(D_{2 n}\right)-2 \Phi\left(D_{2 n-1}\right)-S_{2}\right)\right. \\
\left.\quad \times \Phi\left(D_{2 n}\right)+2 S_{2} \Phi\left(D_{2 n-1}\right)\right] \\
=x\left(\Phi\left(D_{2 n}\right)-2 \Phi\left(D_{2 n-1}\right)\right)\left(\Phi\left(D_{2 n}\right)-S_{2}\right) .
\end{aligned}
$$

Let $\mu_{1}\left(\Phi\left(D_{2 n}\right)-2 \Phi\left(D_{2 n-1}\right)\right)$ and $\mu_{1}\left(\Phi\left(D_{2 n}\right)-S_{2}\right)$ be the largest roots of $\Phi\left(D_{2 n}\right)-2 \Phi\left(D_{2 n-1}\right)=0$ and $\Phi\left(D_{2 n}\right)-S_{2}=$ 0 , respectively. In the proof of Theorem 2 (see [13]), we have shown that the sequence $\mu_{1}\left(\Phi\left(D_{2 n}\right)-2 \Phi\left(D_{2 n-1}\right)\right)$ is strictly increasing by the initial value $\mu_{1}\left(\Phi\left(D_{2}\right)-2 \Phi\left(D_{1}\right)\right)=4.4150$. For any fixed $n$, the second largest $\operatorname{root} \Phi\left(D_{2 n}\right)-2 \Phi\left(D_{2 n-1}\right)=$ 0 is less than 4 .

By Lemmas 5(ii) and 7, we get

$$
\begin{aligned}
& 2 \Phi\left(D_{2 n-1}\right)-S_{2} \\
&=\left(\Phi\left(D_{2 n}\right)-S_{2}\right)-\left(\Phi\left(D_{2 n}\right)-2 \Phi\left(D_{2 n-1}\right)\right) \\
&= P(x) \Phi^{2}\left(D_{n-2}\right)-Q(x) \Phi\left(D_{n-2}\right) \Phi\left(D_{n-3}\right) \\
&+W(x) \Phi^{2}\left(D_{n-3}\right),
\end{aligned}
$$

where

$$
\begin{gathered}
P(x)=2 x^{3}-14 x^{2}+26 x-11>0, \\
Q(x)=4 x^{2}-19 x+16>0, \\
W(x)=2 x-5>0, \\
4 P(x) W(x)-Q^{2}(x)=127 x^{2}-36>0,
\end{gathered}
$$

for $x \geq 4.3$ by derivative.

Then when $n=2,2 \Phi\left(D_{2 n-1}\right)-S_{2}=P(x) \Phi^{2}\left(D_{n-2}\right)>0$. When $n \geq 3$,

$$
\begin{aligned}
& 2 \Phi\left(D_{2 n-1}\right)-S_{2} \\
& \quad \geq(2 \sqrt{P(x) W(x)}-Q(x)) \Phi\left(D_{n-2}\right) \Phi\left(D_{n-3}\right)>0 .
\end{aligned}
$$

If $\mu_{1}\left(\Phi\left(D_{2 n}\right)-2 \Phi\left(D_{2 n-1}\right)\right) \leq \mu_{1}\left(\Phi\left(D_{2 n}\right)-S_{2}\right)$, then we put $x=\mu_{1}\left(\Phi\left(D_{2 n}\right)-S_{2}\right)$ in (17), whose left side is less than and equal to 0 , a contradiction. So, $\mu_{1}\left(\Phi\left(D_{2 n}\right)-2 \Phi\left(D_{2 n-1}\right)\right)>$ $\mu_{1}\left(\Phi\left(D_{2 n}\right)-S_{2}\right)$.

Finally, $\mu_{1}\left(\Phi\left(G_{2 n}(n-1, n)\right)\right)=\mu_{1}\left(\Phi\left(D_{2 n}\right)-2 \Phi\left(D_{2 n-1}\right)\right)$ holds.

Case 2. The unicyclic graph $G_{2 n+1}(n, n)$, where $k=4 n+4(n \geq$ 2 ), is as follows.

Note that

$$
\begin{aligned}
\operatorname{det}(D) \operatorname{det}(B)= & \left(x^{3}-4 x^{2}+4 x-1\right) \Phi^{2}\left(D_{n-2}\right) \\
& -2\left(x^{2}-5 x+2\right) \Phi\left(D_{n-2}\right) \Phi\left(D_{n-3}\right) \\
& +(x-1) \Phi^{2}\left(D_{n-3}\right) \\
\operatorname{det}\left(D^{\prime}\right) \operatorname{det}(B)=( & x-1)^{2} \Phi^{2}\left(D_{n-2}\right)-2(x-1) \\
\times & \Phi\left(D_{n-2}\right) \Phi\left(D_{n-3}\right)+\Phi^{2}\left(D_{n-3}\right), \\
\operatorname{det}(D) \operatorname{det}\left(B^{\prime}\right)= & \left(x^{2}-3 x+1\right) \Phi^{2}\left(D_{n-2}\right) \\
& +\left(x^{2}-4 x+2\right) \Phi\left(D_{n-2}\right) \Phi\left(D_{n-3}\right) \\
& -(x-1) \Phi^{2}\left(D_{n-3}\right) \\
\operatorname{det}\left(D^{\prime}\right) \operatorname{det}\left(B^{\prime}\right)= & (x-1) \Phi^{2}\left(D_{n-2}\right)+(x-2) \Phi\left(D_{n-2}\right) \\
& \times \Phi\left(D_{n-3}\right)-\Phi^{2}\left(D_{n-3}\right) .
\end{aligned}
$$

By (2), (12), and (20), we have

$$
\begin{aligned}
\Phi\left(G_{2 n+1}(n, n)\right) \\
=x \Phi\left(D_{2 n+1}\right) \\
\times\left\{\left(x^{5}-12 x^{4}+50 x^{3}-88 x^{2}+61 x-12\right) \Phi^{2}\left(D_{n-2}\right)\right. \\
\quad-\left(2 x^{4}-20 x^{3}+62 x^{2}-68 x+20\right) \Phi\left(D_{n-2}\right) \\
\left.\quad \times \Phi\left(D_{n-3}\right)+\left(x^{3}-8 x^{2}+16 x-8\right) \Phi^{2}\left(D_{n-3}\right)\right\} \\
+2 x \Phi\left(D_{2 n}\right)\left\{\left(2 x^{3}-10 x^{2}+14 x-4\right) \Phi^{2}\left(D_{n-2}\right)\right. \\
\quad+\left(2 x^{2}-12 x+6\right) \Phi\left(D_{n-2}\right) \Phi\left(D_{n-3}\right) \\
=x\left(U_{1} \Phi\left(D_{2 n+1}\right)+2 U_{2} \Phi\left(D_{2 n}\right)\right) .
\end{aligned}
$$

From Lemmas 5(ii) and 7,

$$
\begin{aligned}
& \Phi\left(D_{2 n+1}\right)-2 \Phi\left(D_{2 n}\right) \\
&= \Phi\left(D_{n+1}\right) \Phi\left(D_{n}\right)-\Phi\left(D_{n}\right) \Phi\left(D_{n-1}\right) \\
&-2\left(\Phi^{2}\left(D_{n}\right)-\Phi^{2}\left(D_{n-1}\right)\right) \\
&=\left(x^{5}-12 x^{4}+52 x^{3}-98 x^{2}+75 x-16\right) \Phi^{2}\left(D_{n-2}\right) \\
&-\left(2 x^{4}-20 x^{3}+66 x^{2}-80 x+26\right)
\end{aligned}
$$




$$
\begin{aligned}
& \times \Phi\left(D_{n-2}\right) \Phi\left(D_{n-3}\right)+\left(x^{3}-8 x^{2}+18 x-10\right) \\
& \times \Phi^{2}\left(D_{n-3}\right) \\
= & U_{1}+U_{2} .
\end{aligned}
$$

So,

$$
\begin{aligned}
\Phi\left(G_{2 n+1}(n, n)\right) \\
=x\left[\left(\Phi\left(D_{2 n+1}\right)-2 \Phi\left(D_{2 n}\right)-U_{2}\right)\right. \\
\left.\quad \times \Phi\left(D_{2 n+1}\right)+2 U_{2} \Phi\left(D_{2 n}\right)\right] \\
=x\left(\Phi\left(D_{2 n+1}\right)-2 \Phi\left(D_{2 n}\right)\right)\left(\Phi\left(D_{2 n+1}\right)-U_{2}\right) .
\end{aligned}
$$

By a similar discussion to that in Case 1 , we have $\mu_{1}\left(\Phi\left(D_{2 n+1}\right)-2 \Phi\left(D_{2 n}\right)\right)>\mu_{1}\left(\Phi\left(D_{2 n+1}\right)-U_{2}\right)$. Thus, $\mu_{1}\left(\Phi\left(G_{2 n+1}(n, n)\right)\right)=\mu_{1}\left(\Phi\left(D_{2 n+1}\right)-2 \Phi\left(D_{2 n}\right)\right)$.

Lemma 9. Give a sequence of Laplacian spectral radii of even unicyclic graphs as follows:

$$
\begin{aligned}
(S): & \mu_{1}\left(G_{(k-2) / 2}(m, n)\right), \\
& \mu_{1}\left(G_{(k-2) / 2}(m-1, n+1)\right), \ldots, \\
& \mu_{1}\left(G_{(k-2) / 2}(0, m+n)\right),
\end{aligned}
$$

where $n \geq 2, m=n-1$, or $m=n$. Then

(i) if $m=n-1$,

when $k=4 n+2$, the items of the sequence $(S)$ are equal to each other; that is,

$$
\begin{aligned}
\mu_{1}\left(G_{2 n}(n-1, n)\right) & =\mu_{1}\left(G_{2 n}(n-2, n+1)\right) \\
& =\cdots=\mu_{1}\left(G_{2 n}(0,2 n-1)\right) ;
\end{aligned}
$$

when $k<4 n+2$, the sequence $(S)$ is strictly decreasing; when $k>4 n+2$, the sequence $(S)$ is strictly increasing;

(ii) if $m=n$,

when $k=4 n+4$, the items of the sequence $(S)$ are equal to each other; that is,

$$
\begin{aligned}
\mu_{1}\left(G_{2 n+1}(n, n)\right) & =\mu_{1}\left(G_{2 n+1}(n-1, n+1)\right) \\
& =\cdots=\mu_{1}\left(G_{2 n+1}(0,2 n)\right) ;
\end{aligned}
$$

when $k<4 n+4$, the sequence $(S)$ is strictly decreasing; when $k>4 n+4$, the sequence $(S)$ is strictly increasing.

Proof. For any $n \geq m \geq 1$, by (7), we have

$$
\begin{aligned}
& \Phi\left(G_{a}(m, n)\right) \\
&= h(x)\left(\Phi\left(D_{m}\right)+\Phi\left(D_{m-1}\right)\right)\left(\Phi\left(D_{n-1}\right)+\Phi\left(D_{n-2}\right)\right) \\
&-i(x)\left(\Phi\left(D_{m-1}\right)+\Phi\left(D_{m-2}\right)\right)\left(\Phi\left(D_{n-1}\right)+\Phi\left(D_{n-2}\right)\right) \\
&-g(x)\left(\Phi\left(D_{m}\right)+\Phi\left(D_{m-1}\right)\right)\left(\Phi\left(D_{n-2}\right)+\Phi\left(D_{n-3}\right)\right) \\
&+k(x)\left(\Phi\left(D_{m-1}\right)+\Phi\left(D_{m-2}\right)\right) \\
& \times\left(\Phi\left(D_{n-2}\right)+\Phi\left(D_{n-3}\right)\right) .
\end{aligned}
$$

From (10), and (27) and Lemma 6,

$$
\begin{aligned}
\Phi\left(G_{(k-2) / 2}(m, n)\right)-\Phi\left(G_{(k-2) / 2}(m-1, n+1)\right) \\
=x\left[(h(x)+k(x)) \Phi\left(D_{n-m-1}\right)-i(x) \Phi\left(D_{n-m}\right)\right. \\
\left.\quad-g(x) \Phi\left(D_{n-m-2}\right)\right] \\
=-x \Phi\left(D_{n-m}\right)\left[x \Phi\left(D_{k-1}\right)-\Phi\left(C_{k}\right)\right. \\
\left.\quad-x \Phi^{2}\left(D_{(k-2) / 2}\right)\right] \\
=-x \Phi\left(D_{n-m}\right) \Phi\left(D_{(k-2) / 2}\right) \\
\quad \times\left[\Phi\left(D_{(k-2) / 2}\right)-2 \Phi\left(D_{(k-4) / 2}\right)\right] .
\end{aligned}
$$

Case $1(m=n-1)$. If $k=4 n+2$, we have $\mu_{1}\left(\Phi\left(G_{2 n}(n-\right.\right.$ $1, n)))=\mu_{1}\left(\Phi\left(D_{2 n}\right)-2 \Phi\left(D_{2 n-1}\right)\right)$ by the previous discussion. From (28), the items of the sequence $(S)$ are equal to each other.

If $k<4 n+2$, by Lemma 3 , then $\mu_{1}\left(G_{(k-2) / 2}(m-i, n+\right.$ i)) $>\mu_{1}\left(G_{2 n}(m-i, n+i)\right)$, where $0 \leq i \leq m$. Note that $\mu_{1}\left(\Phi\left(D_{(k-2) / 2}\right)-2 \Phi\left(D_{(k-4) / 2}\right)\right)<\mu_{1}\left(\Phi\left(D_{2 n}\right)-2 \Phi\left(D_{2 n-1}\right)\right)$; by Lemma 1 and (28), we obtain that the sequence $(S)$ is strictly decreasing.

If $k>4 n+2$, by Lemma 3 , then $\mu_{1}\left(G_{(k-2) / 2}(m-i, n+\right.$ i) $)<\mu_{1}\left(G_{2 n}(m-i, n+i)\right)$, where $0 \leq i \leq m$. Note that $\mu_{1}\left(\Phi\left(D_{(k-2) / 2}\right)-2 \Phi\left(D_{(k-4) / 2}\right)\right)>\mu_{1}\left(\Phi\left(D_{2 n}\right)-2 \Phi\left(D_{2 n-1}\right)\right)$; by Lemma 1 and (28), we obtain that the sequence $(S)$ is strictly increasing.

Case $2(m=n)$. By a similar discussion to that in Case 1, the results of (ii) hold.

Theorem 10. For any $U \in C(n, k)$, that is, $U=C\left(T_{1}, T_{2}\right.$, $\left.\ldots, T_{k}\right)$, if $k$ is even and the number of $T_{i}(1 \leq i \leq k)$ is at most 2 , supposing that $c=\lceil(n-k) / 2\rceil \geq 2$, then

(i) if $n-k=2 c-1$, when $k=4 c+2, \mu_{1}\left(G_{2 c}(c-1, c)\right)=\mu_{1}\left(G_{2 c}(c-2, c+\right.$ 1)) $=\cdots=\mu_{1}\left(G_{2 c}(0, n-k)\right)$ are minimal; when $k<$ $4 c+2, \mu_{1}\left(G_{(k-2) / 2}(0, n-k)\right)$ is the smallest; when $k>$ $4 c+2, \mu_{1}\left(G_{(k-2) / 2}(c-1, c)\right)$ is the smallest;

(ii) if $n-k=2 c$,

when $k=4 c+4, \mu_{1}\left(G_{2 c+1}(c, c)\right)=\mu_{1}\left(G_{2 c+1}(c-1, c+\right.$ $1))=\cdots=\mu_{1}\left(G_{2 c+1}(0, n-k)\right)$ are minimal; when $k<$ $4 n+4, \mu_{1}\left(G_{(k-2) / 2}(0, n-k)\right)$ is the smallest; when $k>$ $4 n+4, \mu_{1}\left(G_{(k-2) / 2}(c, c)\right)$ is the smallest.

Proof. Given any $U \in C(n, k)$, for each $j$, let $T_{j}$ be a rooted tree (with $r_{j}$ as its root) attached to $v_{j}(1 \leq j \leq k)$, where the order of $T_{j}$ is $m_{j}+1$. We assume that all trees but, say, $T_{i}$, are kept fixed, while $T_{i}$ (along with its root) can be changed. Suppose that $T_{i} \neq P_{m_{i}+1}$. Let $v$ be a vertex belonging to $T_{i}$ chosen so that $d(v)>2$ and that $d\left(v, r_{i}\right)$ (the distance between $v$ and $\left.r_{i}\right)$ is the largest. By Lemma 4 , the Laplacian spectral radius is strictly decreasing when two hanging paths at $v$ are replaced by a new hanging path (i.e., the length of the new hanging path is the sum of the lengths of two hanging paths at $v$ ). If 
the same is repeated for other hanging paths to $v$, we get one path attached at $v$ (its central vertex is identified with $v$ ) whose size is equal to the sum of the lengths of the aforementioned paths. Let $w$ be a vertex in $T_{i}$, adjacent to $v$ and belonging to the (unique) path between $r_{i}$ and $v$. Note also that $d\left(w, r_{i}\right)=$ $d\left(v, r_{i}\right)-1$. By repeating the same procedure (for any other vertex as $v$ ), we arrive at $G_{1}$, where the rooted tree $T_{i}$ becomes a path $P_{m_{i}+1}$, so that $\mu_{1}\left(G_{1}\right)<\mu_{1}(U)$.

By the same way as with other rooted trees, we arrive at $G_{2}$, where every rooted tree $T_{j} \cong P_{m_{j}+1}(1 \leq j \leq k)$; that is, $G_{2} \cong P\left(m_{1}, m_{2}, \ldots, m_{k}\right)$. By Lemma $4, \mu_{1}\left(G_{2}\right)<\mu_{1}(U)$ holds.

If there are at most two trees attached to the cycle $C_{k}$, by the previous discussion, we get that $P\left(m_{1}, m_{2}, \ldots, m_{k}\right)$ has small Laplacian spectral radius among $C(n, k)$, where two $m_{i}(1 \leq i \leq k)$ are nonzero at most. Applying Lemma 8 repeatedly, we know that $G_{(k-2) / 2}(a, b)$ has the smaller Laplacian spectral radius, where $a+b=n-k$. From Lemma 9, the result holds.

Remark 11. When $n-k=2$, that is, $c=1$, from Theorem 2 and its proof (see [13]), we get the same result as Theorem 10.

\section{Acknowledgments}

The author would like to express his sincere gratitude to the referees and the handing editor for the very careful reading of the paper and for the insightful comments and valuable suggestions, which led to improving this paper. The research of the author is supported by the National Natural Science Foundation of China (no. 61303020) and the National Natural Science Foundation of Shanxi Province (no. 2012011019-2).

\section{References}

[1] D. Cvetkovic, M. Doob, and H. Sachs, Spectra of Graphs, Academic Press, New York, NY, USA, 1976.

[2] W. N. Anderson and T. D. Morley, "Eigenvalues of the Laplacian of araph," Linear and Multi-Linear Algebra, vol. 18, pp. 141-145, 1985.

[3] K. C. Das, “The Laplacian spectrum of a graph," Computers and Mathematics with Applications, vol. 48, no. 5-6, pp. 715-724, 2004.

[4] R. Grone and R. Merris, "The Laplacian spectrum of a graph," SIAM Journal on Discrete Mathematics, vol. 7, pp. 221-229, 1994.

[5] J. S. Li and X. D. Zhang, "A new upper bound for eigenvalues of the Laplacian matrix of a graph," Linear Algebra and Its Applications, vol. 265, pp. 93-100, 1997.

[6] J. S. Li and X. D. Zhang, "On the Laplacian eigenvalues of a graph," Linear Algebra and Its Applications, vol. 285, pp. 305307, 1998.

[7] R. Merris, "Laplacian matrices of graphs: a survey, Linear Algebra and Its Applications, vol. 197-198, pp. 143-176, 1994.

[8] S. G. Guo, "The largest eigenvalues of Laplacian matrices of unicyclic graphs," Applied Mathematics A, vol. 16, no. 2, pp. 131-135, 2001.

[9] H. X. Zhang, "The largest Laplacian spectral radius of unicyclic graphs with fixed diameter," Journal of Applied Mathematics. In press.
[10] J. Guo, "The Laplacian spectral radius of a graph under perturbation," Computers and Mathematics with Applications, vol. 54, no. 5, pp. 709-720, 2007.

[11] J. M. Guo, "The effect on the Laplacian spectral radius of a graph by adding or grafting edges," Linear Algebra and Its Applications, vol. 413, no. 1, pp. 59-71, 2006.

[12] J. Guo, "A conjecture on the algebraic connectivity of connected graphs with fixed girth," Discrete Mathematics, vol. 308, no. 23, pp. 5702-5711, 2008.

[13] H. X. Zhang, "Ordering some even-unicyclic graphs by their Laplacian spectral radii” (Chinese), Journal of Dalian University of Technology. In press. 


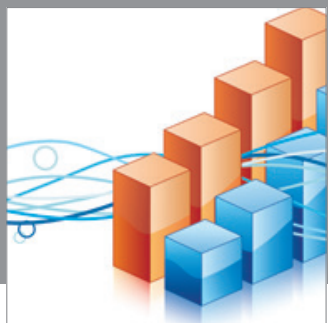

Advances in

Operations Research

mansans

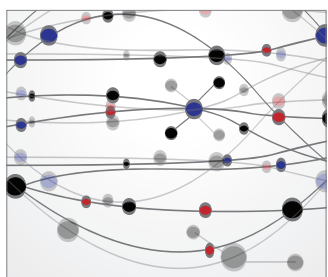

The Scientific World Journal
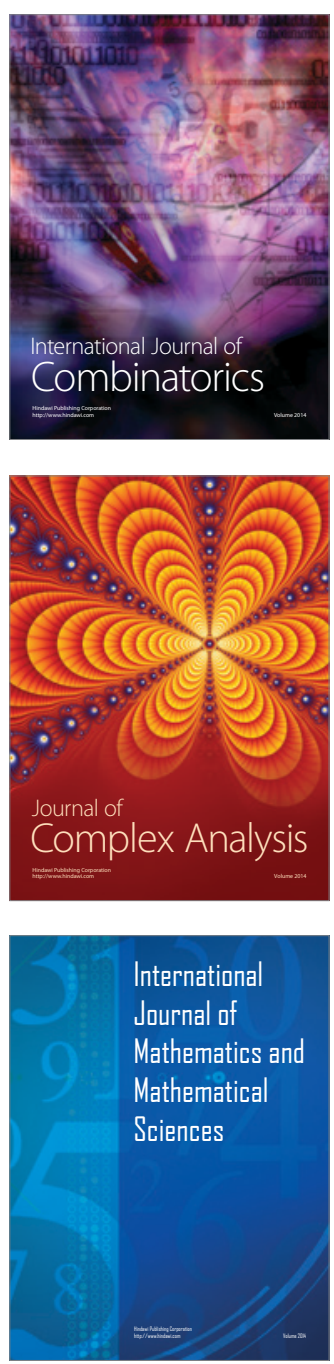
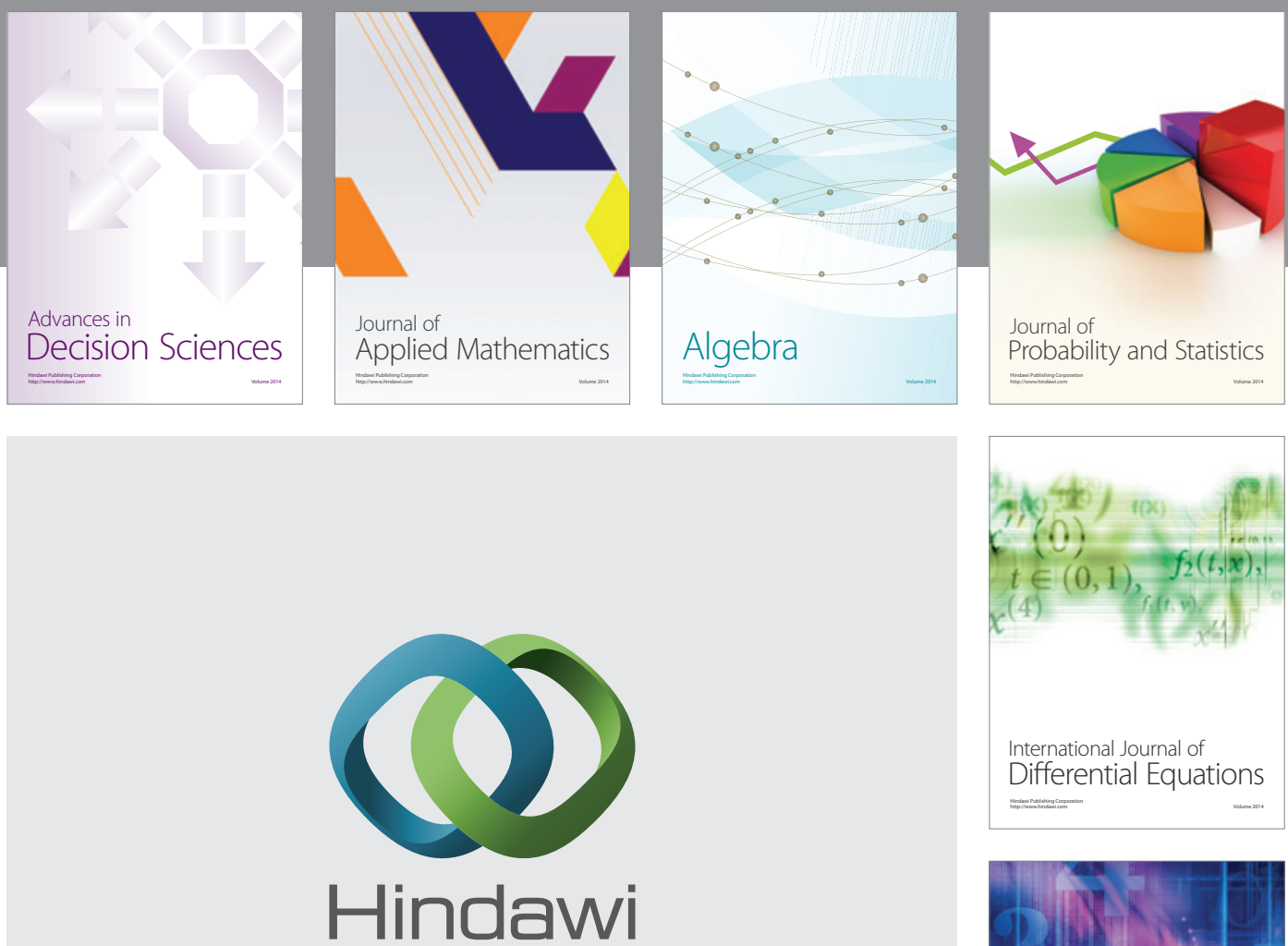

Submit your manuscripts at http://www.hindawi.com
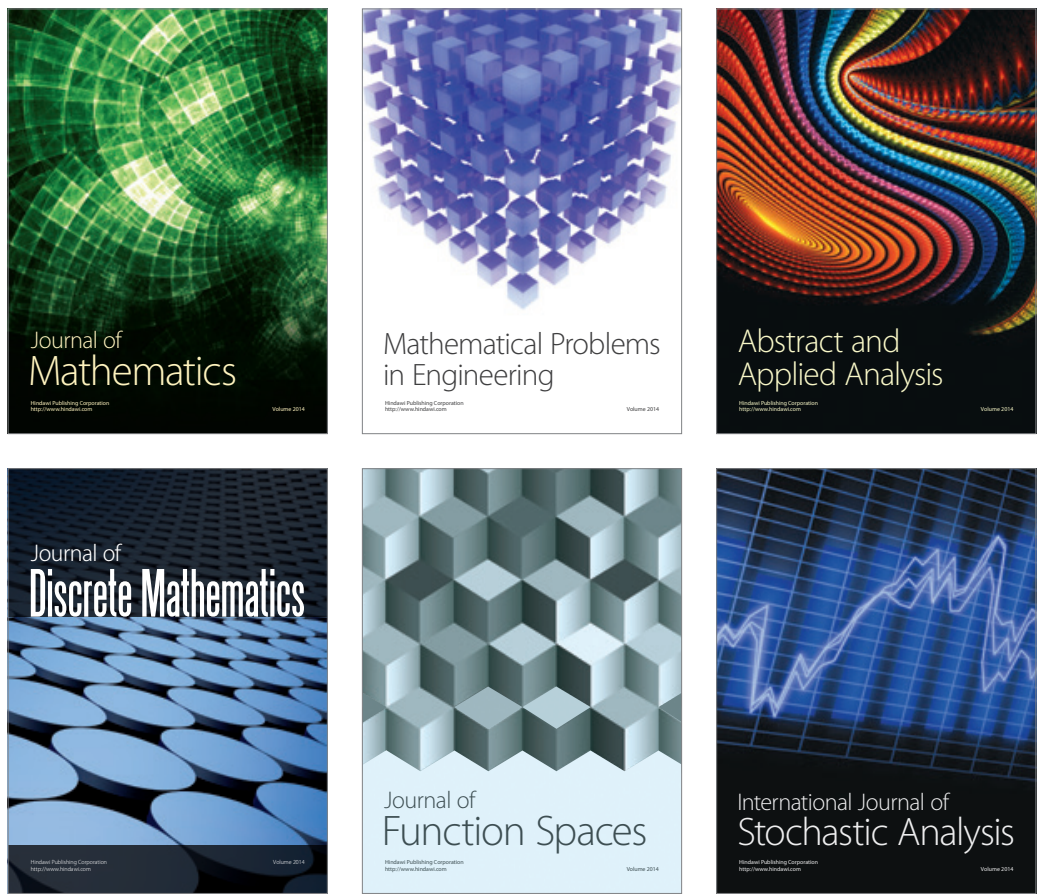

Journal of

Function Spaces

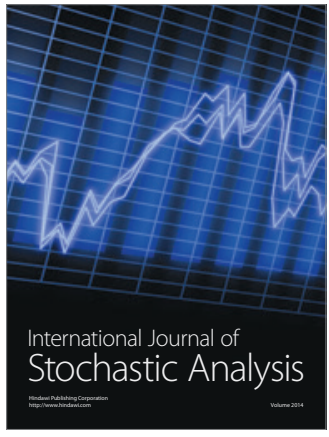

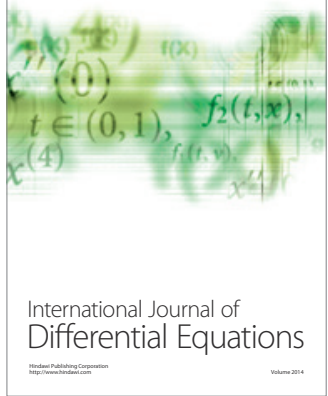
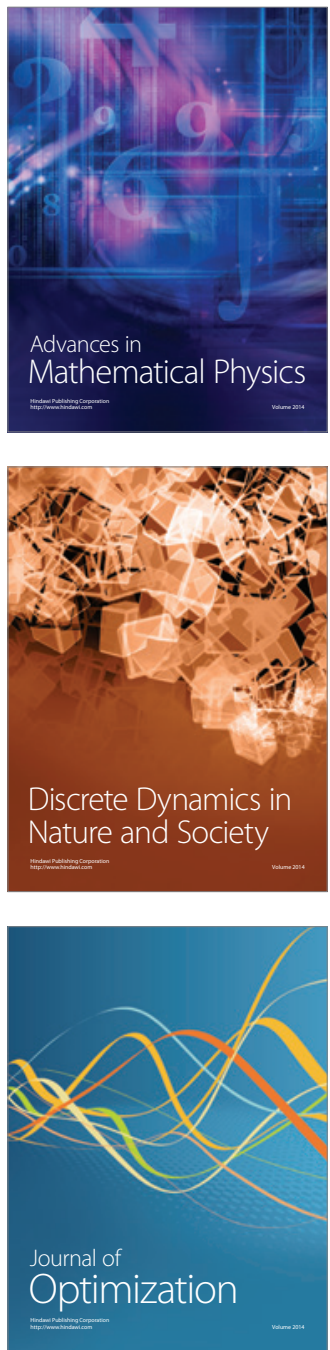